\title{
Enabling 4-Lane Based 400 G Client-Side Transmission Links with MultiCAP Modulation
}

\author{
Anna Tatarczak, ${ }^{1}$ Miguel Iglesias Olmedo, ${ }^{1,2}$ Tianjian Zuo, ${ }^{3}$ Jose Estaran, ${ }^{1}$ \\ Jesper Bevensee Jensen, ${ }^{1}$ Xiaogeng $\mathrm{Xu},{ }^{3}$ and Idelfonso Tafur Monroy ${ }^{1}$ \\ ${ }^{1}$ DTU Fotonik, Technical University of Denmark, Building 343, 2800 Kongens Lyngby, Denmark \\ ${ }^{2}$ Optics Division, Royal Institute of Technology, Electrum 229, 16440 Kista, Sweden \\ ${ }^{3}$ Transmission Technology Research Department, Huawei Technologies Co., Ltd., Shenzhen 518129, China
}

Correspondence should be addressed to Anna Tatarczak; atat@fotonik.dtu.dk

Received 9 May 2015; Revised 15 July 2015; Accepted 22 July 2015

Academic Editor: José Luís Santos

Copyright (C) 2015 Anna Tatarczak et al. This is an open access article distributed under the Creative Commons Attribution License, which permits unrestricted use, distribution, and reproduction in any medium, provided the original work is properly cited.

\begin{abstract}
We propose a uniform solution for a future client-side $400 \mathrm{G}$ Ethernet standard based on MultiCAP advanced modulation format, intensity modulation, and direct detection. It employs 4 local area networks-wavelength division multiplexing (LAN-WDM) lanes in $1300 \mathrm{~nm}$ wavelength band and parallel optics links based on vertical cavity surface emitting lasers (VCSELs) in $850 \mathrm{~nm}$ wavelength band. Total bit rate of $432 \mathrm{Gbps}$ is transmitted over unamplified $20 \mathrm{~km}$ standard single mode fiber link and over $40 \mathrm{~km}$ link with semiconductor optical amplifier. $70.4 \mathrm{~Gb} / \mathrm{s}$ transmission over $100 \mathrm{~m}$ of OM3 multimode fiber using off-the-shelf $850 \mathrm{~nm}$ VCSEL with $10.1 \mathrm{GHz} 3 \mathrm{~dB}$ bandwidth is demonstrated indicating the feasibility of achieving $100 \mathrm{~Gb} / \mathrm{s}$ per lane with a single $25 \mathrm{GHz} \mathrm{VCSEL}$. In this review paper we introduce and present in one place the benefits of MultiCAP as versatile scheme for use in a number of client-side scenarios: short range, long range, and extended range.
\end{abstract}

\section{Introduction}

Ever growing video-rich Ethernet traffic on the client-side optical networks calls for high-speed, cost-effective optical transport data links. Standardization of client-side optical data links is critical to ensure compatibility and interoperability of telecom and datacom equipment from different vendors. As depicted in Figure 1, the IEEE standardization body classifies the client-side links in three categories: short range (SR), long range (LR), and extended range (ER). SR links, which cover up to $100 \mathrm{~m}$, are usually employed in data centers and central offices. LR links cover up to $20 \mathrm{~km}$ and are typically used to privately connect buildings of the same company or institution. ER links cover up to $40 \mathrm{~km}$ and are typically used to provide connectivity to customer-premises equipment (CPE) and for metro applications. The current work of the 400 Gbps Ethernet Study Group [1] and the wider research community focuses on these three scenarios [2]. An optical intensity modulation/direct detection (IM/DD) link offering $400 \mathrm{Gbps}$ capacity with use of advanced modulation formats is an attractive and easily adaptable solution for client-side links, such as inter- and intradata center interconnects.

The client-side links presented in this work are based on the multiband and multilevel approach to carrierless amplitude phase (CAP) modulation, MultiCAP [3]. In this review paper we demonstrate the flexibility of the IM/DD MultiCAP based solutions for a SR $100 \mathrm{~m}$ link [4], a LR $20 \mathrm{~km}$ link, and an ER $40 \mathrm{~km}$ link [5]. A SR client-side link that achieves error-free $65.7 \mathrm{Gbps}$ over a $100 \mathrm{~m}$ multimode fiber (MMF) OM3 using an $850 \mathrm{~nm}$ vertical cavity surface-emitting laser (VCSEL) is presented. Furthermore, two IM/DD LAN-WDM $432 \mathrm{Gbps}$ links are described: an unamplified $20 \mathrm{~km}$ link for the LR scenario and semiconductor optical amplifier (SOA) based $40 \mathrm{~km}$ link for the ER scenario. Four-lane LANWDM with 108 Gbps per lane is obtained using 4 externally modulated lasers (EMLs) in the O-band.

Figure 2 summarizes the capacity per lane reported at the considered transmission distances for several modulation formats. The short range (SR) area of Figure 2 shows the highest error-free bit rates achieved for $850 \mathrm{~nm}$ vertical cavity surface-emitting laser (VCSEL) based links. Bit rate of 


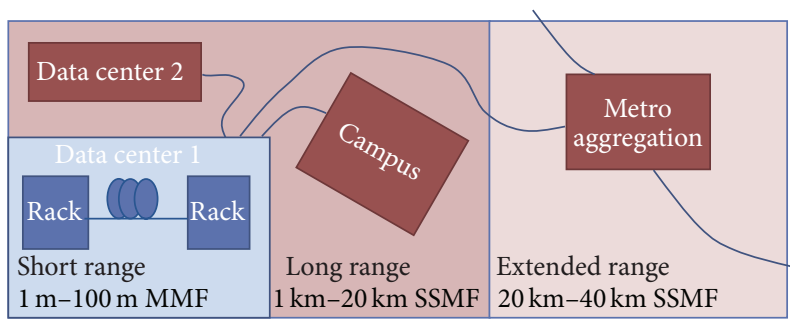

FIGURE 1: Scenario of client-side optical transmission links for short range (SR), long range (LR), and extended range (ER).

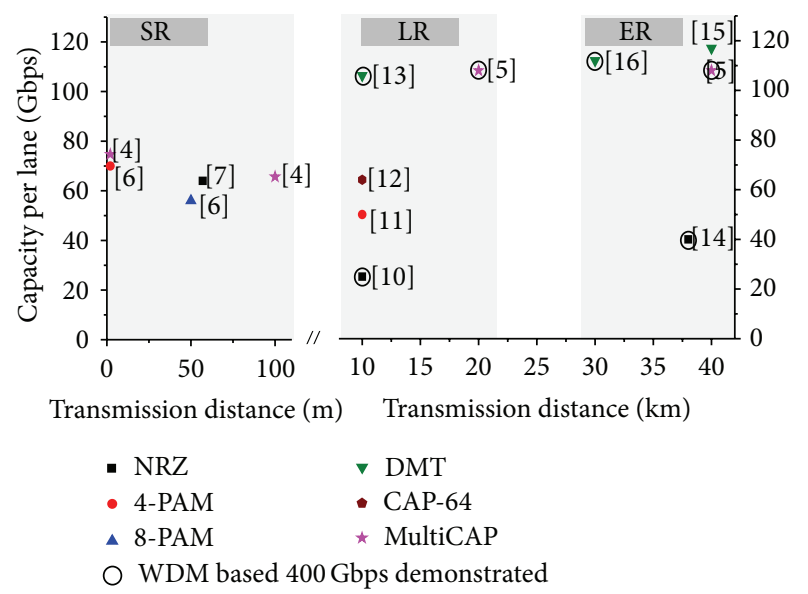

FIGURE 2: State-of-the-art summary; capacity per lane versus transmitted distance for the following modulation formats: Nonreturn to Zero (NRZ), pulse amplitude modulation (PAM), discrete multitone (DMT), carrierless amplitude phase (CAP), and multiband carrierless amplitude phase (MultiCAP), demonstrated in literature approaches to $400 \mathrm{Gbps}$ systems based on IM/DD WDM are indicated by the black circle.

70 Gbps over $2 \mathrm{~m}$ OM4 MMF was achieved using 4-level pulse amplitude modulation (4-PAM) [6], 64 Gbps over $57 \mathrm{~m}$ OM2 using Nonreturn to Zero (NRZ) [7], and 56 Gbps over $50 \mathrm{~m}$ OM4 using 8-PAM [6]. All of these require very fast electrical interfaces and suffer from low tolerance to modal dispersion compared to pass-band modulation formats [8]. Using discrete multitone (DMT) at an $850 \mathrm{~nm}$ window enabled a high transmission distance of $500 \mathrm{~m}$ MMF with a bit rate of 30-Gbps [9].

The MultiCAP solution presented in this paper achieves error-free $65.7 \mathrm{Gbps}$ over $100 \mathrm{~m}$ and $74.7 \mathrm{Gbps}$ over $1 \mathrm{~m}$ using an $850 \mathrm{~nm}$ VCSEL with a bandwidth of $10.1 \mathrm{GHz}$. This solution has the prospect of achieving 100 Gbps over $100 \mathrm{~m}$ MMF with emerging $25 \mathrm{GHz} 850 \mathrm{~nm}$ VCSELs. It overcomes both electrical and optical bandwidth limitations towards single lane 100 Gbps active optical cable (AOC) and employs cost efficient $850 \mathrm{~nm}$ MMF technologies. The $400 \mathrm{GE}$ standard requirement can thus be met by employing parallel optical lanes.

The client-side links of long range (LR) and extended range (ER) are expected to meet the $400 \mathrm{Gbps}$ capacity by using advanced modulation formats in combination with wavelength division multiplexing (WDM) [1]. The higher the capacity per lane, the lower the number of WDM lanes and therefore the number of transceivers. The LR and ER areas in Figure 2 show the highest capacities per lane reported in O-band for different modulation formats. In the LR of $10 \mathrm{~km}$, NRZ coding enables 25 Gbps [10], 4-PAM 50 Gbps [11], CAP64QAM 60 Gbps [12], and DMT 106 Gbps [13]. MultiCAP achieves $108 \mathrm{Gbps}$ per lane with $20 \mathrm{~km}$ reach [5]. In the ER of $40 \mathrm{~km}$, NRZ coding allows $40 \mathrm{Gbps}$ per lane [14]. Beyond $100 \mathrm{Gbit} / \mathrm{s}$ per lane for ER is reached by DMT modulation [15] and MultiCAP [5]. The DD/IM WDM-based 400 Gbps systems were demonstrated as feasible in several of the cited works (indicated in Figure 2). Eight lanes $\times 40$ Gbps [14] or 16 lanes $\times 25$-Gbps [10] were used to reach $400 \mathrm{Gbps}$ with NRZ coding. A four-lane LAN-WDM $400 \mathrm{Gbps}$ solution was demonstrated using DMT over $30 \mathrm{~km} \mathrm{[16]} \mathrm{and} \mathrm{MultiCAP}$ over $40 \mathrm{~km}$ standard single mode fiber (SSMF) [5]. Both of them assume the $7 \%$ FEC overhead.

The main contribution of this paper is the overview of a uniform MultiCAP based solution for short, long, and extended range client-side links. In all of these scenarios the same implementation scheme can be used. We review the previously presented experimental results focusing on the implementation similarities for different client-side scenarios. We include detailed description of the performed experiments. Moreover, we present the first full description of the used equalizer that was used in previous reported experiments. Having a uniform modulation format in different links types, lengths, and different wavelength bands will not only allow for interoperability between kinds of equipment from different vendors but also reduce the cost and complexity for the clients. In this way a newly developed client's link can leverage the already existing implementation of different link type. We show that using the same transceiver's structure and equalization technique allows satisfying the $400 \mathrm{GE}$ capacity requirement in SR, LR, and ER. MultiCAP advanced modulation format is combined with parallel optics in SR and with WDM in LR and ER. This easily applicable solution enables a simple upgrade from $100 \mathrm{Gbps}$ to $400 \mathrm{Gbps}$ in both $850 \mathrm{~nm}$ MM links and $1310 \mathrm{~nm}$ SM links. In the context of $400 \mathrm{Gbps}$ Ethernet standardization we demonstrate that a MultiCAP based solution is feasible and worth considering for SR, LR, and ER.

\section{Methods}

Figure 3 depicts the experimental setup for all of the considered transmission scenarios. At the transmitter side, 5 effective number of bits (ENOB) $64 \mathrm{GSa} / \mathrm{s}$ digital-to-analog converter (DAC) is used to generate MultiCAP signal. The transmitter consists of a linear amplifier and a laser. $850 \mathrm{~nm}$ VCSEL is used and in SR EMLs are used in LR and ER scenarios. The channel consists of $100 \mathrm{~m}$ MMF for SR and $20 \mathrm{~km}$ of SSMF for LR. ER scenario consists of $40 \mathrm{~km} \mathrm{SSMF}$ and an SOA at the receiver. The receiver consists of a photodiode, transimpedance amplifiers (TIAs), and a digital storage oscilloscope (DSO). The $400 \mathrm{Gbps}$ standard requirement in the SR multimode scenario is expected to be fulfilled by parallel optics. Therefore, for the SR scenario we verify only one lane. In the LR and ER scenarios the expected solution to reach $400 \mathrm{Gbps}$ is WDM. Hence, in the experimental 

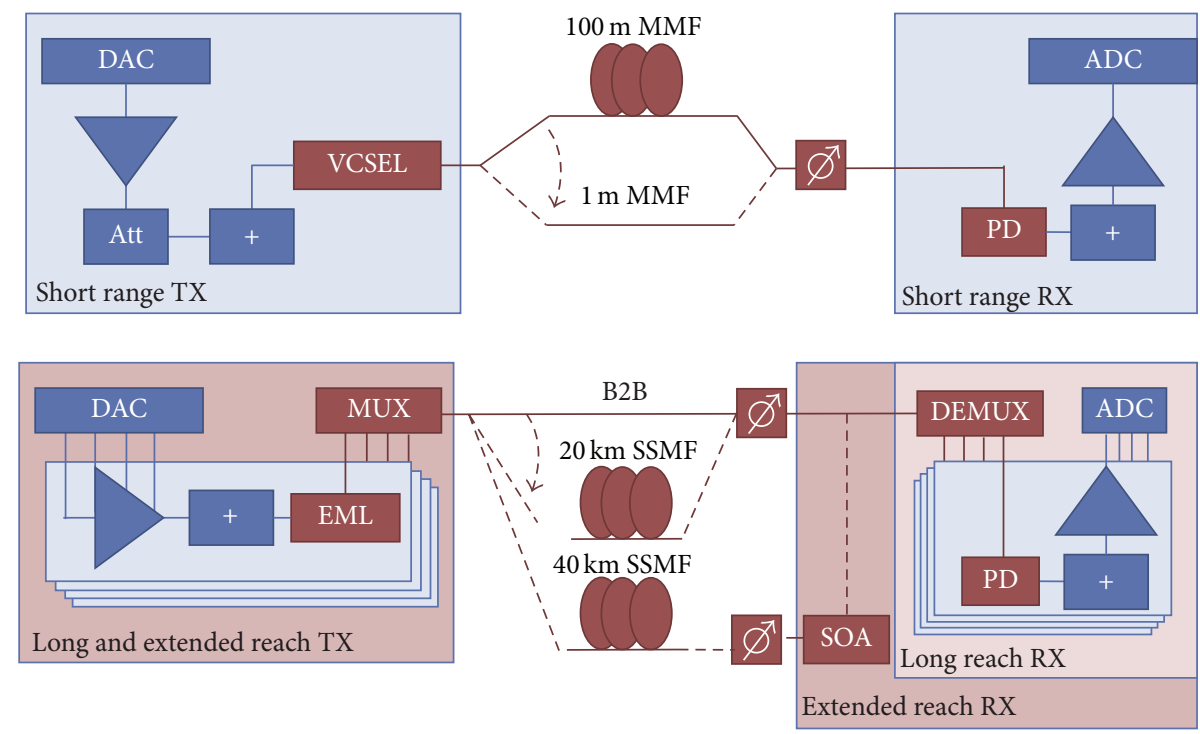

FIGURE 3: Experimental setup of the short range (SR) scenario employs digital-to-analog converter (DAC), attenuator (Att), vertical cavity surface-emitting laser (VCSEL), multimode fiber (MMF), photodiode (PD), and analog-to-digital converter (ADC); setups for long range (LR) and extended range (ER) additionally employ externally modulated laser (EML), multiplexer (MUX), standard single mode fiber (SSMF), semiconductor optical amplifier (SOA), and demultiplexer (DEMUX).

TABLE 1: Modulation order per band for different bit rates.

\begin{tabular}{|c|c|c|c|c|c|c|c|c|c|}
\hline Number & Scenario & Bit rate & Baud rate per band & B1 & B2 & B3 & $\mathrm{B} 4$ & B5 & B6 \\
\hline 1 & SR & 70.4 Gbps & 3.4 Gbaud & 32 & 32 & 16 & 8 & 8 & 4 \\
\hline 2 & SR & 80 Gbps & 3.4 Gbaud & 64 & 32 & 32 & 16 & 8 & 4 \\
\hline 3 & LR and ER & 108 Gbps & 4 Gbaud & 64 & 64 & 32 & 16 & 16 & 4 \\
\hline
\end{tabular}

verification of the LR and ER setup, four independent channels of DAC are used to drive four parallel lanes of WDM transmitter. Additionally, a WDM transmitter includes the WDM multiplexer and the receiver a WDM demultiplexer.

2.1. Signal Generation. The signals are generated by a 4output $64 \mathrm{GSa} / \mathrm{s}$ digital-to-analog converter (DAC) with 5 ENOB. For signal generation, we choose a 6-band configuration of MultiCAP [3] with different modulation orders per band which result in different bit rates. Table 1 presents three configurations and Figures 4(c)-4(e) depict the corresponding electrical spectra. Each MultiCAP band is constructed from a pseudorandom bit sequence (PRBS) of $2^{13}-1$ bits and delivers a baud rate as described in Table 1 . The total number of transmitted symbols is 49146. MultiCAP symbols are generated by upsampling to 16 samples per symbol and subsequent CAP filtering. Upsampling factor is an integer multiple of baud rate of each subband. The upsampling procedure is explained in detail in [3]. The CAP filters are realized as finite impulse response (FIR) with a length of 20 symbols for SR scenario and 30 symbols for LR and ER scenarios. A roll-off coefficient of 0.05 was used at the transmitter. At the receiver, time inverted versions of the CAP filters (roll off $=0.09$ ) are used to recover the symbol constellations. We use the MultiCAP features of power and bit loading. The constellation and power level for each band differs and is chosen empirically to best fit the signal-to-noise ratio (SNR) of the specific frequency band. The bands' configuration and power choice depend on the frequency response of the overall system.

The frequency response of SR system is presented in Figure 4(a). A $3 \mathrm{~dB}$ bandwidth of $10.1 \mathrm{GHz}$, a $10 \mathrm{~dB}$ bandwidth of $17 \mathrm{GHz}$, and a $20 \mathrm{~dB}$ bandwidth of $20.1 \mathrm{GHz}$ are measured. This frequency response allows for the first and the second MultiCAP bands configurations presented in Table 1. First configuration shown in Table 1 and in Figure 4(c) enables a total throughput of $70.4 \mathrm{Gbps}(65.7 \mathrm{Gbps}$ after $7 \%$ overhead forward error correction (FEC) decoding), whereas the second configuration shown in Figure 4(d) enables 80 Gbps (74.7 Gbps after 7\% FEC). In these two cases, 6 MultiCAP bands occupied the bandwidth of $21 \mathrm{GHz}$. The frequency response of optical back-to-back for both LR and ER systems is presented in Figure 4(b). A 3-dB bandwidth of $8.90 \mathrm{GHz}$, a $10-\mathrm{dB}$ bandwidth of $17.35 \mathrm{GHz}$, and a $20-\mathrm{dB}$ bandwidth of $24 \mathrm{GHz}$ are observed. The bandwidth in this case is effectively limited by the bandwidth of the DAC used. This response allowed for implementing the last band configuration from Table 1 presented in Figure 4(e). This configuration enabled throughput of $108 \mathrm{Gbps}$ (100.9 Gbps after 7\% FEC). Bandwidth of $26 \mathrm{GHz}$ has been used for MultiCAP bands.

2.2. Short Range. A commercially available $850 \mathrm{~nm}$ VCSEL is used in the SR scenario. Figure 5 shows the LIV curves and the optical spectrum measured for the VCSEL. The center 


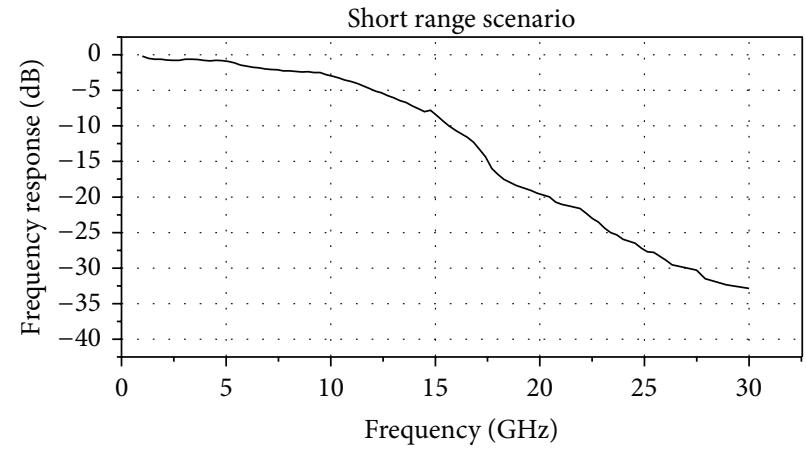

$-S_{21}$

(a)

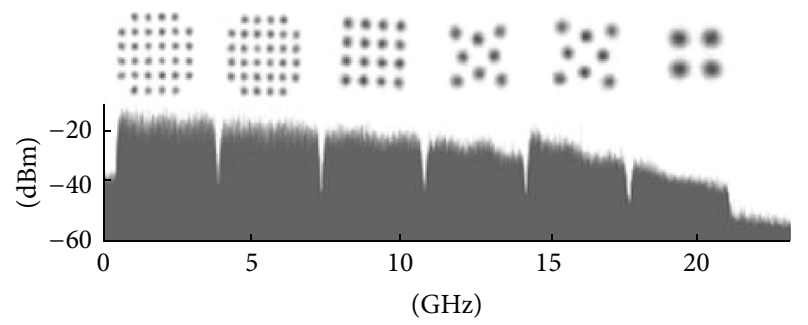

(c)

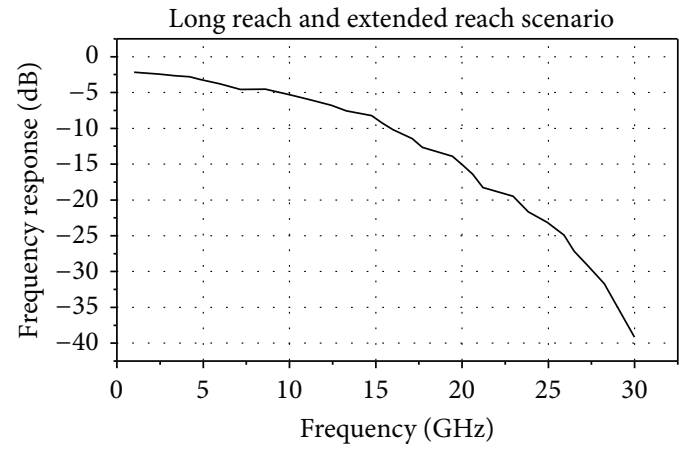

(b)

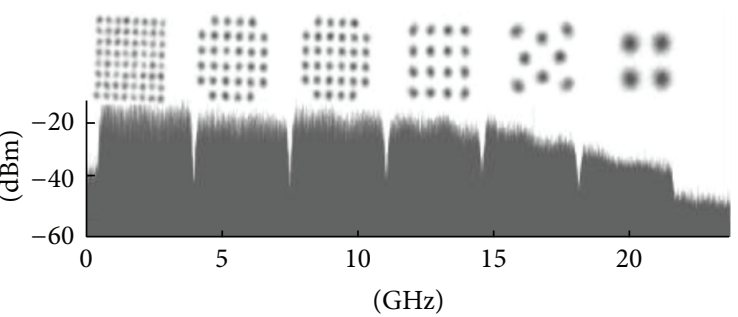

(d)

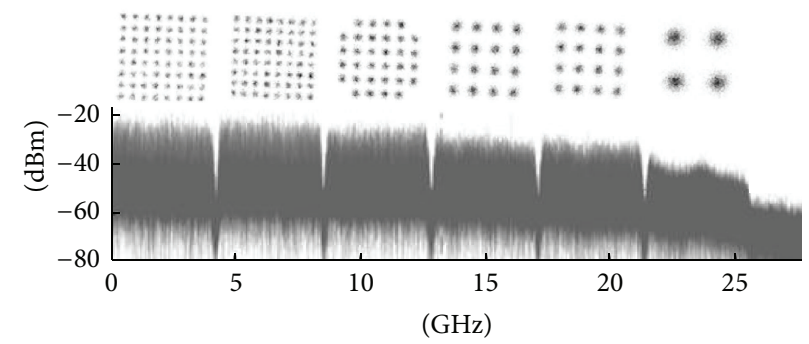

(e)

FIGURE 4: (a) Frequency response measured for short range (SR) scenario end-to-end link; (b) frequency response measured for the optical back-to-back of long range (LR) and extended range (ER) scenarios. Received electrical MultiCAP spectrum and corresponding constellations transmitted in each MultiCAP band for (c) short range (SR) scenario with total bit rate $70.4 \mathrm{Gbps}$; (d) short range (SR) scenario with total bit rate $80 \mathrm{Gbps}$; (e) long range (LR) and extended range (ER) scenarios with total bit rate $108 \mathrm{Gbps}$.
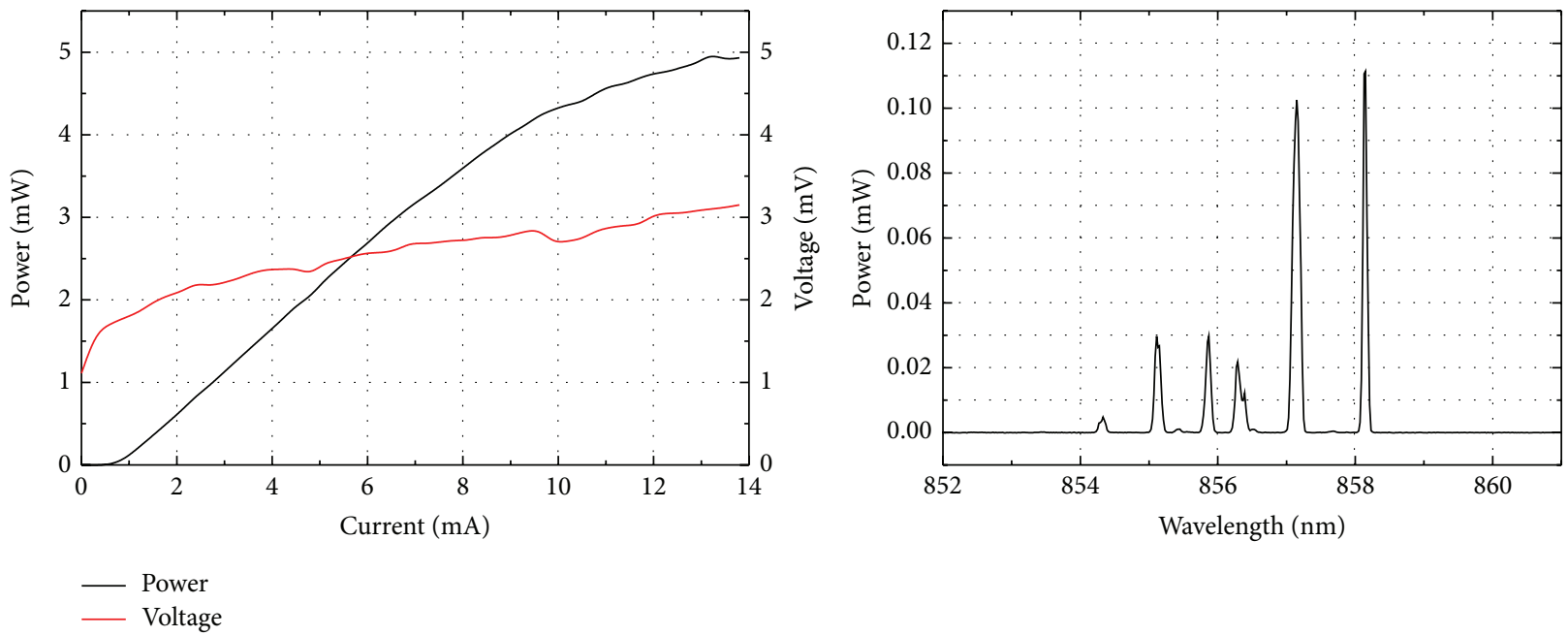

FIGURE 5: Characterization of the VCSEL used in the short range scenario; LIV curve measured at the room temperature; optical spectrum measured at bias current of $8 \mathrm{~mA}$. 

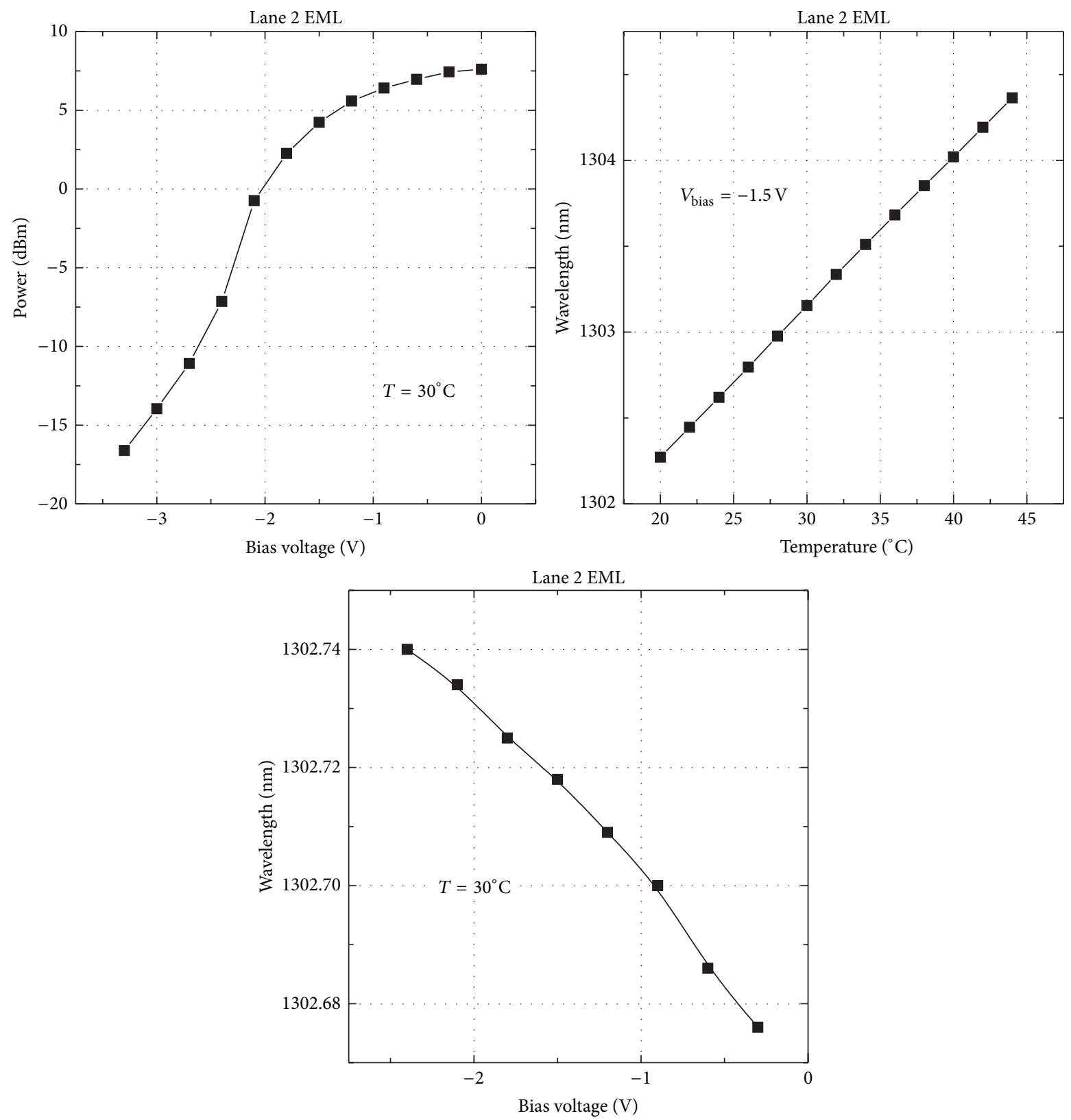

FIGURE 6: Characterization of the EML of Lane 2; power versus bias current measured at $T=30^{\circ}$; wavelength versus temperature measured at bias voltage of $-1.5 \mathrm{~V}$; wavelength versus bias voltage measured at $T=30^{\circ}$.

frequency of the VCSELs' spectrum at $8 \mathrm{~mA}$ bias is $857.2 \mathrm{~nm}$. The DAC output is amplified to a $1.2 \mathrm{Vp}$-p signal that is used to drive the VCSEL biased at $8 \mathrm{~mA}$. An optical power of $6 \mathrm{dBm}$ is launched into $100 \mathrm{~m}$ of OM3 compliant MMF, with a total link loss of $0.5 \mathrm{~dB}$. The signal is photodetected with an $850 \mathrm{~nm}$ photodiode reverse biased at $4 \mathrm{~V}$. The signal is then amplified to a Vp-p of $1 \mathrm{~V}$ and digitally stored with an $80 \mathrm{GSa} / \mathrm{s}$ DSO with a resolution of 8 bits.

2.3. Long Range and Extended Range. The signals generated by a 4-output DAC are decorrelated with delay lines. The laser source used in these scenarios is EML. The bias voltage and temperature characteristics of the EML employed in
Lane 2 are presented in Figure 6. The EML's bias voltage is $-1.5 \mathrm{~V}$ and the MultiCAP signal has a CMOS compatible peak-to-peak voltage of $2.5 \mathrm{Vp}$-p. The center wavelengths of the EMLs in Lanes 0 to 3 are $1294 \mathrm{~nm}, 1299 \mathrm{~nm}, 1303 \mathrm{~nm}$, and $1308 \mathrm{~nm}$. In order to keep the wavelengths stable, temperature control is applied. The output power of the EML in the tested lane is $6 \mathrm{dBm}$. Average output power of the EMLs ranges from $4 \mathrm{dBm}$ to $6 \mathrm{dBm}$.

The optical signals are combined in a LAN-WDM multiplexer (MUX) with a channel spacing of $800 \mathrm{GHz}$ (G.694.1 compliant) and transmitted over $20 \mathrm{~km}$ or $40 \mathrm{~km}$ (G.652 compliant) SSMF links. MUX introduces $0.6 \mathrm{~dB}$ of insertion loss. The span losses are $7 \mathrm{~dB}$ and $14 \mathrm{~dB}$, respectively. For the 
TABLE 2: Equalizer's performance in terms of BER as compared to the nonequalized system measured for three different signal-tonoise ratio (SNR) settings; compared systems: nonequalized system with a standard multimodulus algorithm (MMA) equalization and with decision directed (DD) $K$-means equalizer.

\begin{tabular}{lccc}
\hline $\begin{array}{c}\text { SNR } \\
(\mathrm{dB})\end{array}$ & $\begin{array}{c}\text { BER } \\
\text { nonequalized } \\
\text { system }\end{array}$ & $\begin{array}{c}\text { BER } \\
\text { MMA } \\
\text { equalizer }\end{array}$ & $\begin{array}{c}\text { BER } \\
\text { DD } K \text {-means } \\
\text { equalizer }\end{array}$ \\
\hline 20.6 & $1.89 \cdot 10^{-2}$ & $3.83 \cdot 10^{-3}$ & $8.67 \cdot 10^{-4}$ \\
19.5 & $2.23 \cdot 10^{-2}$ & $7.66 \cdot 10^{-3}$ & $3.9 \cdot 10^{-3}$ \\
18.2 & $3.67 \cdot 10^{-2}$ & $1.96 \cdot 10^{-2}$ & $1.48 \cdot 10^{-2}$ \\
\hline
\end{tabular}

$40 \mathrm{~km}$ transmission case, a semiconductor optical amplifier (SOA) with a noise figure (NF) of $6.5 \mathrm{~dB}$ is employed at the receiver, before demultiplexing. At the receiver side the signal is demultiplexed by a LAN-WDM demultiplexer (DEMUX), received by a photodiode (PD), and amplified by a transimpedance amplifier (TIA). DEMUX introduces $0.9 \mathrm{~dB}$ of insertion loss. All of the components are 100GBASE-LR4 and ER4 compatible.

2.4. Demodulation and Equalization. The receiver consists of several digital signal processing (DSP) blocks which are implemented in Matlab environment. CAP filtering, signal downsampling, phase offset removal, and signal normalization are performed as explained in [3]. Additionally, we implement an adaptive frequency domain equalization to mitigate linear impairments. The described adaptive decision directed (DD) equalization algorithm minimizes the received constellation cluster size and quantization noise.
We define the reference constellation by the centroids found using $K$-means algorithm which groups the received data in the clusters [17]. This reference constellation initializes the described DD equalization algorithm. Clusters' means are the points of reference (starting decision).

We use an iterative equalizer where in every iteration the following steps are performed: first, the error is calculated based on the Euclidean distance from the closest centroid as in a least mean square (LMS) equalizer:

$$
\varepsilon(n)=\min \|\mathbb{C}-y(n)\|,
$$

where $\mathbb{C}$ denotes all centroids of the reference constellation and $y(n)$ is the received signal sample. For equalization we use $T / 2$ fractionally spaced FIR filter with 12 taps determined empirically. The taps coefficients of the DD equalizer are updated according to the following equation:

$$
h(n+1)=h(n)+\mu(n) \cdot \varepsilon(n) \cdot y(n)^{*},
$$

where $h(n)$ is the equalizer coefficient, $\mu(n)$ is the step size initialized as $7.5 \cdot 10^{-4}$, and $y(n)^{*}$ is the complex conjugate $y(n)$. Secondly, the received signal is passed through the equalizer. Finally, the iterative process reestimates the centroids of the equalized constellation and the described steps are repeated. It was experimentally determined that 2 iterations result in satisfactory equalization and further iterations do not show the performance improvement. To assure a faster convergence we implement the variable step in DD. The step size is updated in the following manner [18]:

$$
\begin{aligned}
\mu(n+1) & =\frac{\mu(n)}{1+\lambda \mu(n)|\varepsilon(n)|^{2}}, \\
\lambda= & \begin{cases}1, & \text { if } n=0 \\
0, & \text { if } \operatorname{sgn}(\operatorname{Re}\{\varepsilon(n)\})=\operatorname{sgn}(\operatorname{Re}\{\varepsilon(n-1)\}), \operatorname{sgn}(\operatorname{Im}\{\varepsilon(n)\})=\operatorname{sgn}(\operatorname{Im}\{\varepsilon(n-1)\}) \\
1, & \text { otherwise, }\end{cases}
\end{aligned}
$$

where sgn denotes a sign function.

In order to quantify the improvement due to using an equalizer, we calculate BER for the equalized and nonequalized system for three different SNR values. Moreover, we present BER calculated for the system with a standard frequency domain equalizer, namely, multimodulus algorithm (MMA). Table 2 summarizes the BERs for all equalization and SNR scenarios. Decision directed (DD) $K$-means equalizer improves the performance in terms of BER in all three SNR scenarios. At SNR of $20.6 \mathrm{~dB}$ using an equalizer allows for the improvement of 0.0181 in terms of BER. In the following sections all of the presented results are equalized using DD $K$-means algorithm.

After the signal is equalized, the EVM is calculated and BER is computed. In order to calculate bit error rate (BER), the received demodulated signal is cross-correlated with the transmitted signal and the errors are counted.

\section{Experimental Results}

Figure 7 shows the measured BER curves for SR scenario. We define the sensitivity at a BER of the hard decision FEC code at $7 \%$ overhead. For the reported system it is $4.5 \cdot 10^{-3}$ [19]. Thereby, we can observe sensitivities of 2.1, 4.7, and $5.4 \mathrm{dBm}$ for experimentally obtained $70.4 \mathrm{Gbps}$ over $1 \mathrm{~m}, 70.4 \mathrm{Gbps}$ over $100 \mathrm{~m}$, and $80 \mathrm{Gbps}$ over $1 \mathrm{~m}$, respectively. The measured transmission penalty after $100 \mathrm{~m}$ MMF is $2.5 \mathrm{~dB}$.

For LR scenario, per lane received bit error ratio (BER) back-to-back (B2B) and after $20 \mathrm{~km}$ SSMF transmission (no SOA) of the received signal is plotted in Figure $8(\mathrm{a})$. The 


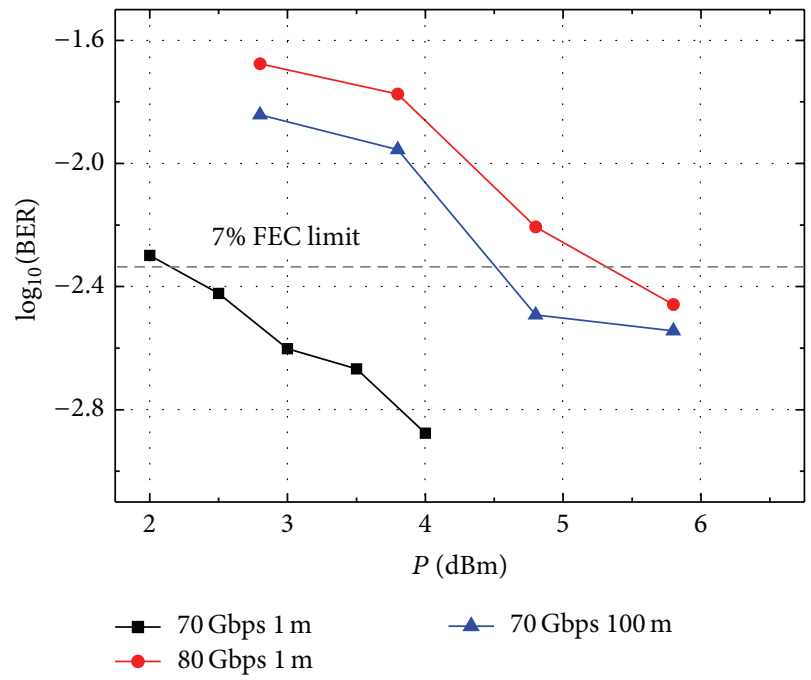

FIGURE 7: BER versus received optical power (ROP) for B2B and $100 \mathrm{~m}$ transmission for $70 \mathrm{Gbps}$ MultiCAP configuration and B2B for $80 \mathrm{Gbps}$ configuration.

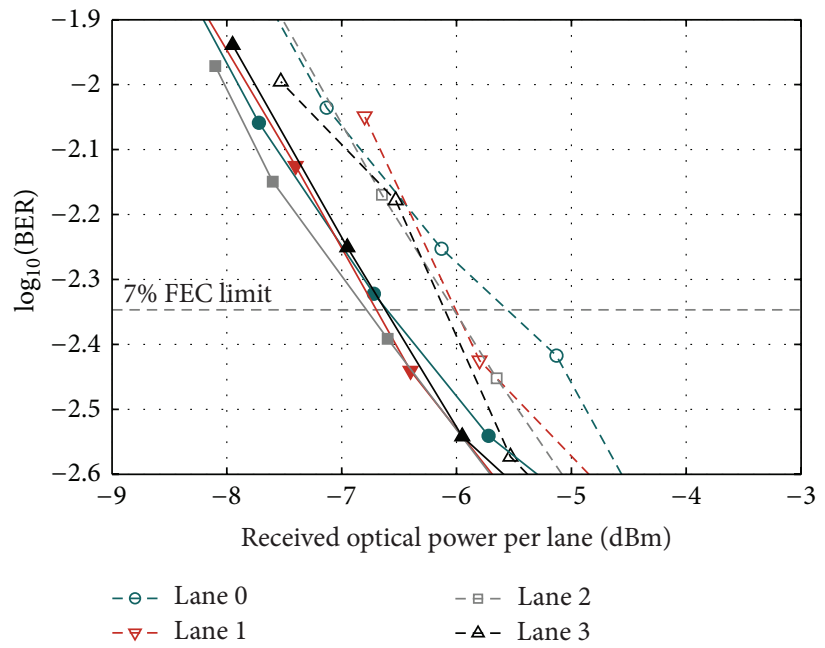

(a)

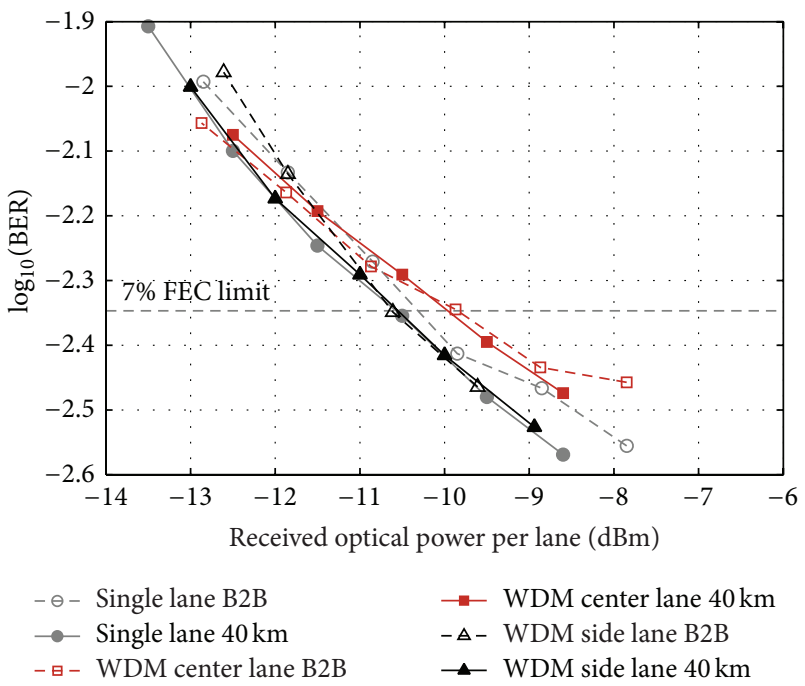

(b)

FIGURE 8: (a) BER versus received optical power (ROP) for B2B and $20 \mathrm{~km}$ transmission for 4 lanes; (b) BER versus ROP for the data transmitted in the single lane (Lane 2) and for the data transmitted in all lines (BER curves for center lane, Lane 2, and side lane, Lane 3); ROP measured before SOA; the total bitrate of WDM system is $432 \mathrm{Gbps}$.

received optical power is measured before the MUX. For all LAN-WDM lanes, BERs are below the 7\% hard decision FEC limit, and no error floor is observed within the tested power range. Receiver sensitivity at the FEC limit is $-6.0 \mathrm{dBm} \mathrm{B} 2 \mathrm{~B}$ and $-6.6 \mathrm{dBm}$ after transmission. No transmission power penalty is observed. The results for ER scenario are presented in Figure 8(b). Received BER of a center lane and a side lane is plotted B2B and after $40 \mathrm{~km}$ SSMF transmission with all 4 LAN-WDM lanes simultaneously amplified by a single SOA before demultiplexing. Received optical power per channel is measured before the SOA. For comparison, the BER of a single lane (remaining three lanes switched off) is included in the graph. All LAN-WDM lanes were received with a BER below the FEC limit after $40 \mathrm{~km}$ SSMF transmission with a worst-case receiver sensitivity of $-9.9 \mathrm{dBm}$. Presence of neighboring channels in the link does not introduce penalty in the $20 \mathrm{~km}$ scenario. In case of $40 \mathrm{~km}$ scenario, we observe a $0.5 \mathrm{~dB}$ power penalty for the center lanes in the 4-lane case due to interlane modulation in the SOA. In both scenarios no penalty is observed in the side lanes.

In the results presented, BER is an average of the BERs in all MultiCAP bands.

Finally, the power budget calculation is evaluated in Table 3. For the SR scenario, the optical output power measured at the output of the VCSEL is $6 \mathrm{dBm}$. The sensitivity at $7 \%$ FEC limit for 70 Gbps $1 \mathrm{~m}$ transmissions is equal to $2.4 \mathrm{dBm}$. Therefore power budget for this scenario is equal to 3.6 dB. In the LR and ER scenarios, the optical output power 
TABLE 3: System power budget.

\begin{tabular}{lccc}
\hline $\begin{array}{l}\text { Transmission } \\
\text { link type }\end{array}$ & $\begin{array}{l}\text { Output } \\
\text { power }\end{array}$ & $\begin{array}{c}\text { Sensitivity @ } \\
\text { FEC limit }\end{array}$ & $\begin{array}{c}\text { System } \\
\text { power } \\
\text { budget }\end{array}$ \\
\hline $\begin{array}{l}\text { sr } 70 \mathrm{Gbps} \\
\text { LR } 20 \mathrm{~km}\end{array}$ & $6 \mathrm{dBm}$ & $2.4 \mathrm{dBm}$ & $\mathbf{3 . 6 \mathrm { dB }}$ \\
$\begin{array}{l}\text { unamplified } \\
\begin{array}{l}\text { ER } 40 \mathrm{~km} \text { SOA } \\
\text { amplified }\end{array}\end{array}$ & $5.4 \mathrm{dBm}$ & $-6.6 \mathrm{dBm}$ & $\mathbf{1 2 . 6 \mathbf { d B }}$ \\
\hline
\end{tabular}

per lane is equal to $5.4 \mathrm{dBm}$. It is measured after transmitter and hence after MUX. The worst receiver sensitivity is $-6.6 \mathrm{dBm}$ at FEC limit in case of $20 \mathrm{~km}$ transmission link with no amplification. Therefore the power budget of this link is $12.6 \mathrm{~dB}$. In case of $40 \mathrm{~km}$ transmission with SOA based amplification, the worst receiver sensitivity is $-9.9 \mathrm{dBm}$ at the FEC limit. Therefore for the amplified $40 \mathrm{~km}$ link, the power budget is $15.9 \mathrm{~dB}$. The given receiver sensitivity is based on ROP measured before receiver: before PD in SR case, before DEMUX in LR case, and before SOA in ER case.

The SR scenario represents a solution for an active optical cable for data centers. In terms of power budget, the margin is necessary only for the components heating up and aging. In case of LR and ER, the calculated margin of $5.6 \mathrm{~dB}$ and $1.9 \mathrm{~dB}$ is sufficient for client-side links.

\section{Discussion}

In the results presented for SR, a steep roll-off of the VCSEL's frequency response reduces the achievable capacity. We use the bit loading and power loading features of MultiCAP to overcome those limitations, at the cost of worse sensitivity. As a consequence, increasing the capacity from $70 \mathrm{Gbps}$ to $80 \mathrm{Gbps}$ introduces the $3.1 \mathrm{~dB}$ penalty in sensitivity as shown in Figure 7. The bandwidth of the existing VCSELs is not sufficient to support $100 \mathrm{Gbps}$ per lane. With the proposed MultiCAP scheme, the emerging 25 Gbps VCSELs are expected to satisfy the bandwidth requirement.

The performance of the EMLs used in LR and ER is satisfactory to obtain $100 \mathrm{Gbps}$ after FEC per lane. Moreover, the local area network-wavelength division multiplexing (LAN-WDM) is proved to introduce negligible penalty both for $20 \mathrm{~km}$ and for $40 \mathrm{~km}$ link. The power budget calculation indicates the maturity of the solution, which allows for link losses of $12.6 \mathrm{~dB}$ and $15.9 \mathrm{~dB}$ in LR and ER, respectively.

The clear difference in performance and achievable capacity between SR and LR, ER scenarios is attributed to the system bandwidth. Even though the $3 \mathrm{~dB}$ and $10 \mathrm{~dB}$ bandwidths are similar for both systems, the $20 \mathrm{~dB}$ bandwidth varies by $5 \mathrm{~dB}$. For this reason, the MultiCAP in SR is recoverable when it occupies up to $21 \mathrm{GHz}$ while the LR and ER signal is possible to recover when it occupies $26 \mathrm{GHz}$ (Figure 4). The last band in all three scenarios is highly suppressed, but thanks to the power loading and bit loading features of MultiCAP, the information in the last band is also possible to recover if it carries QPSK.
The proposed approach for $400 \mathrm{Gbit} / \mathrm{s}$ client-side transmission links using MultiCAP modulation format represents an easily applicable solution that is robust, simple, and flexible in upgrading from $100 \mathrm{Gbit} / \mathrm{s}$ to $400 \mathrm{Gbit} / \mathrm{s}$ while operating at the O-band LAN-WDM wavelengths. Moreover, we present applicability of the MultiCAP solution in the SR multimode (MM) links. We expect that with higher bandwidth of the upcoming $850 \mathrm{~nm}$ VCSELs this solution will enable $100 \mathrm{Gbps}$ per lane and $400 \mathrm{Gbps}$ using parallel optics. This technology potentially provides a bridge for gray optics approach to client-side, inter- and intradata centers, access, and metro segments.

\section{Conclusions}

We present a uniform MultiCAP based solution for short range (SR) MM links, long range (LR) $20 \mathrm{~km}$ single mode (SM) links, and extended range (ER) $40 \mathrm{~km}$ SM links. The advantageous feature of MultiCAP approach of being able to assign parallel electrical interfaces of smaller bandwidth into different frequency bands overcomes both electrical and optical bandwidth limitations and eases the DSP pipelining. Its pass-band nature and multiband structure allow optimal usage of the available bandwidth maximizing obtainable capacity. In the SR scenario, we have achieved record belowFEC bit rate transmission of $65.7 \mathrm{Gbps}$ over $100 \mathrm{~m}$ and 74.7 Gbps over $1 \mathrm{~m}$ for $850 \mathrm{~nm}$ MMF data links. For upcoming $400 \mathrm{GE}$ standard long range and extended range criteria, we present a MultiCAP LAN-WDM 400 Gbps solution which uses only commercial optical components from 100GBASELR4 and ER4. $432 \mathrm{Gbit} / \mathrm{s}$ MultiCAP signals are transmitted over $20 \mathrm{~km}$ SSMF without amplification and over $40 \mathrm{~km}$ SSMF with SOA. Interchannel mixing in the $40 \mathrm{~km}$ link and in SOA is proven to be negligible for a MultiCAP IM/DD LAN-WDM system. The proposed MultiCAP approach is a robust and flexible scheme, which can cover most of the client-side scenarios, including inter- and intradata centers and up to $40 \mathrm{~km}$ client-side links.

\section{Conflict of Interests}

The authors declare that there is no conflict of interests regarding the publication of this paper.

\section{References}

[1] IEEE 400 Gb/s Ethernet Study Group Meeting Materials, 2014.

[2] J. D'Ambrosia and P. Mooney, “400 Gb/s ethernet: why now?" Whitepaper of Ethernet Alliance, 2013.

[3] M. I. Olmedo, T. Zuo, J. B. Jensen et al., "Multiband carrierless amplitude phase modulation for high capacity optical data links," Journal of Lightwave Technology, vol. 32, no. 4, pp. 798804, 2014.

[4] M. I. Olmedo, A. Tatarczak, T. Zuo, J. Estaran, X. Xu, and I. T. Monroy, "Towards 100 Gbps over $100 \mathrm{~m}$ MMF using a $850 \mathrm{~nm}$ VCSEL," in Proceedings of the Optical Fiber Communications Conference and Exhibition (OFC '14), pp. 1-4, March 2014.

[5] T. Zuo, A. Tatarczak, M. Olmedo et al., "O-band 400 Gbit/s client side optical transmission link," in Proceedings of the 
Optical Fiber Communications Conference and Exhibition (OFC '14), pp. 1-3, San Francisco, Calif, USA, March 2014.

[6] K. Szczerba, P. Westbergh, M. Karlsson, P. A. Andrekson, and A. Larsson, "70 Gbps 4-PAM and 56 Gbps 8-PAM using an $850 \mathrm{~nm}$ VCSEL," in Proceedings of the European Conference on Optical Communication (ECOC '14), September 2014.

[7] D. Kuchta, A. V. Rylyakov, C. L. Schow et al., "64 Gb/s transmission over $57 \mathrm{~m}$ MMF using an NRZ modulated $850 \mathrm{~nm}$ VCSEL," in Proceedings of the Optical Fiber Communications Conference and Exhibition (OFC '14), pp. 1-3, Optical Society of America, March 2014.

[8] L. Raddatz and I. H. White, "Overcoming the modal bandwidth limitation of multimode fiber by using passband modulation," IEEE Photonics Technology Letters, vol. 11, no. 2, pp. 266-268, 1999.

[9] S. Lee, F. Breyer, S. Randel, D. Cardenas, H. van den Boom, and A. Koonen, "Discrete multitone modulation for highspeed data transmission over multimode fibers using 850-nm VCSEL," in Proceedings of the Conference on Optical Fiber Communication-Incudes Post Deadline Papers (OFC '09), pp. 1-3, IEEE, San Diego, Calif, USA, March 2009.

[10] Y. Doi, T. Ohyama, T. Yoshimatsu, S. Soma, and M. Oguma, "400GbE demonstration utilizing 100GbE optical sub-assemblies and cyclic arrayed waveguide gratings," in Proceedings of the Optical Fiber Communications Conference and Exhibition (OFC '14), pp. 1-3, San Francisco, Calif, USA, March 2014.

[11] W. Kobayashi, T. Fujisawa, S. Kanazawa, and H. Sanjoh, "25 Gbaud/s 4-PAM (50 Gbit/s) modulation and $10 \mathrm{~km}$ SMF transmission with $1.3 \mu \mathrm{m}$ InGaAlAs-based DML," Electronics Letters, vol. 50, no. 4, pp. 299-300, 2014.

[12] J. Zhang, X. Li, Y. Xia et al., "60-Gb/s CAP-64QAM Transmission using DML with direct detection and digital equalization," in Proceedings of the Optical Fiber Communication Conference and Exposition and the National Fiber Optic Engineers Conference (OFC/NFOEC '14), IEEE, March 2014.

[13] T. Chan, I.-C. Lu, J. Chen, W. Way, and T. Chan, "400-Gb/s transmission over 10-km SSMF using discrete multitone and 1.3-mm EMLs," IEEE Photonics Technology Letters, vol. 26, no. 16, pp. 1657-1660, 2014.

[14] J. P. Turkiewicz and H. de Waardt, "Low complexity up to 400$\mathrm{Gb} / \mathrm{s}$ transmission in the 1310-nm wavelength domain," IEEE Photonics Technology Letters, vol. 24, no. 11, pp. 942-944, 2012.

[15] W. Yan, L. Li, B. Liu et al., " $80 \mathrm{~km}$ IMDD transmission for 100 Gb/s per lane enabled by DMT and nonlinearity management," in Proceedings of the Optical Fiber Communication Conference, p. M2I.4, Optical Society of America, San Francisco, Calif, USA, March 2014.

[16] T. Tanaka, M. Nishihara, T. Takahara et al., "Experimental demonstration of 448-Gbps+ DMT transmission over $30-\mathrm{km}$ SMF," in Proceedings of the Optical Fiber Communications Conference and Exhibition (OFC '14), pp. 1-3, March 2014.

[17] N. G. Gonzalez, D. Zibar, X. Yu, and I. T. Monroy, "Optical phase-modulated radio-over-fiber links with K-means algorithm for digital demodulation of 8PSK subcarrier multiplexed signals," in Proceedings of the Conference on Optical Fiber Communication, Collocated National Fiber Optic Engineers Conference (OFC/NFOEC '10), pp. 1-3, March 2010.

[18] D. Ashmawy, K. Banovic, E. Abdel-Raheem, M. Youssif, H. Mansour, and M. Mohanna, "Joint MCMA and DD blind equalization algorithm with variable-step size," in Proceedings of the IEEE International Conference on Electro/Information Technology (EIT '09), pp. 174-177, June 2009.
[19] F. Chang, K. Onohara, and T. Mizuochi, "Forward error correction for $100 \mathrm{G}$ transport networks," IEEE Communications Magazine, vol. 48, no. 3, pp. S48-S55, 2010. 

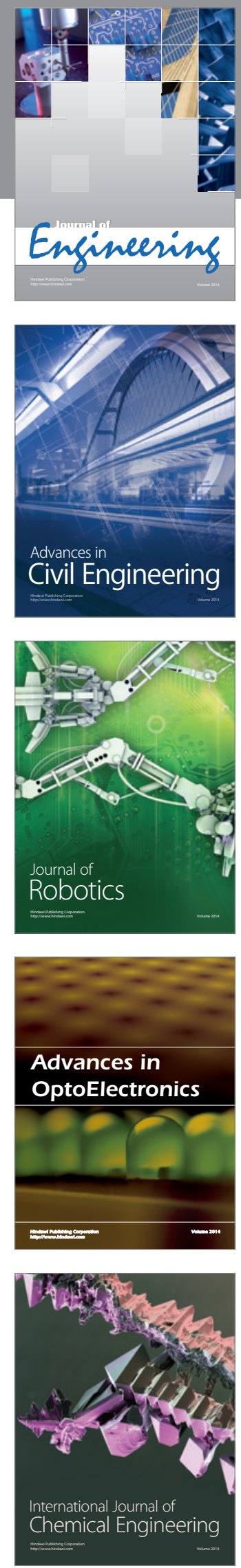

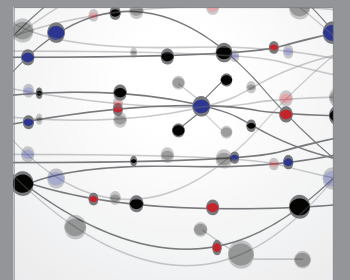

The Scientific World Journal
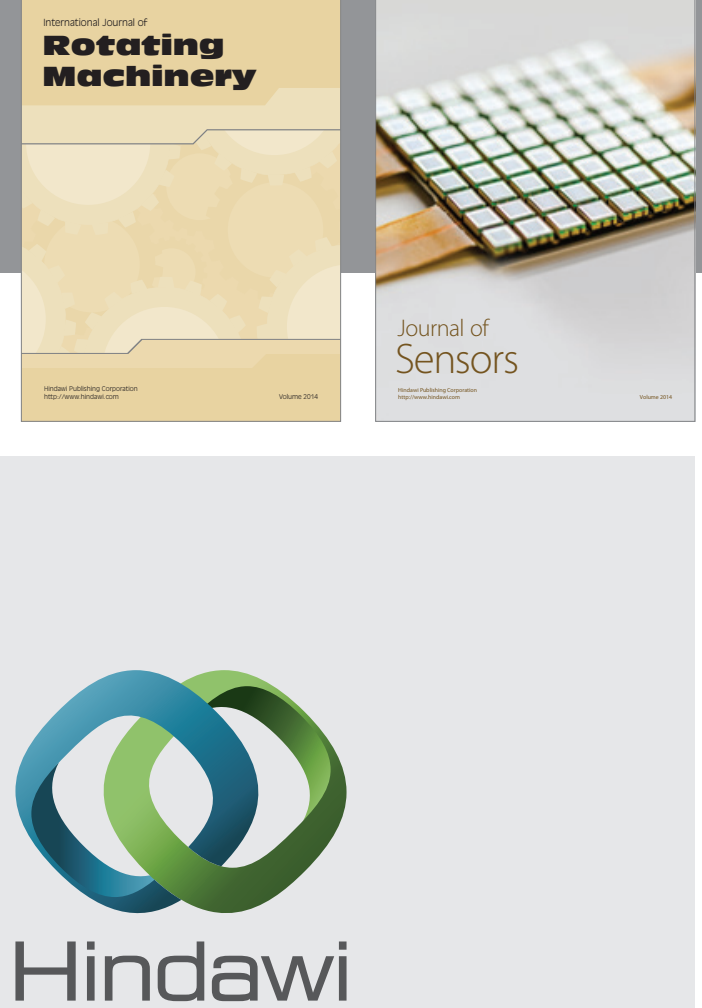

Submit your manuscripts at http://www.hindawi.com
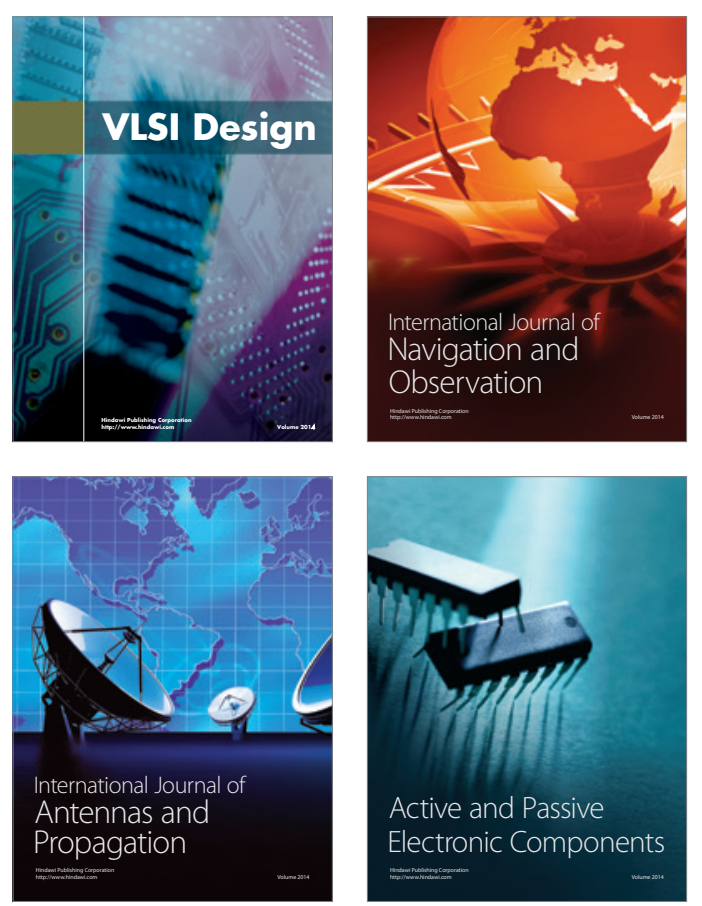
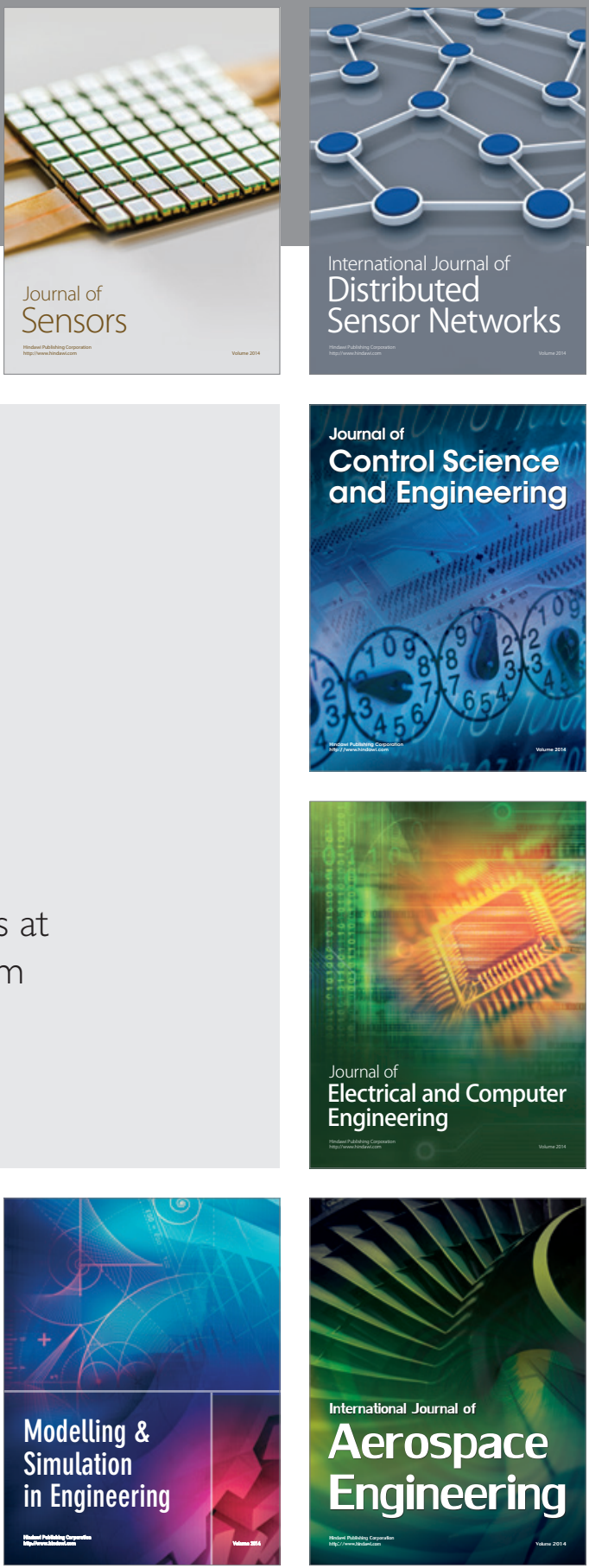

Journal of

Control Science

and Engineering
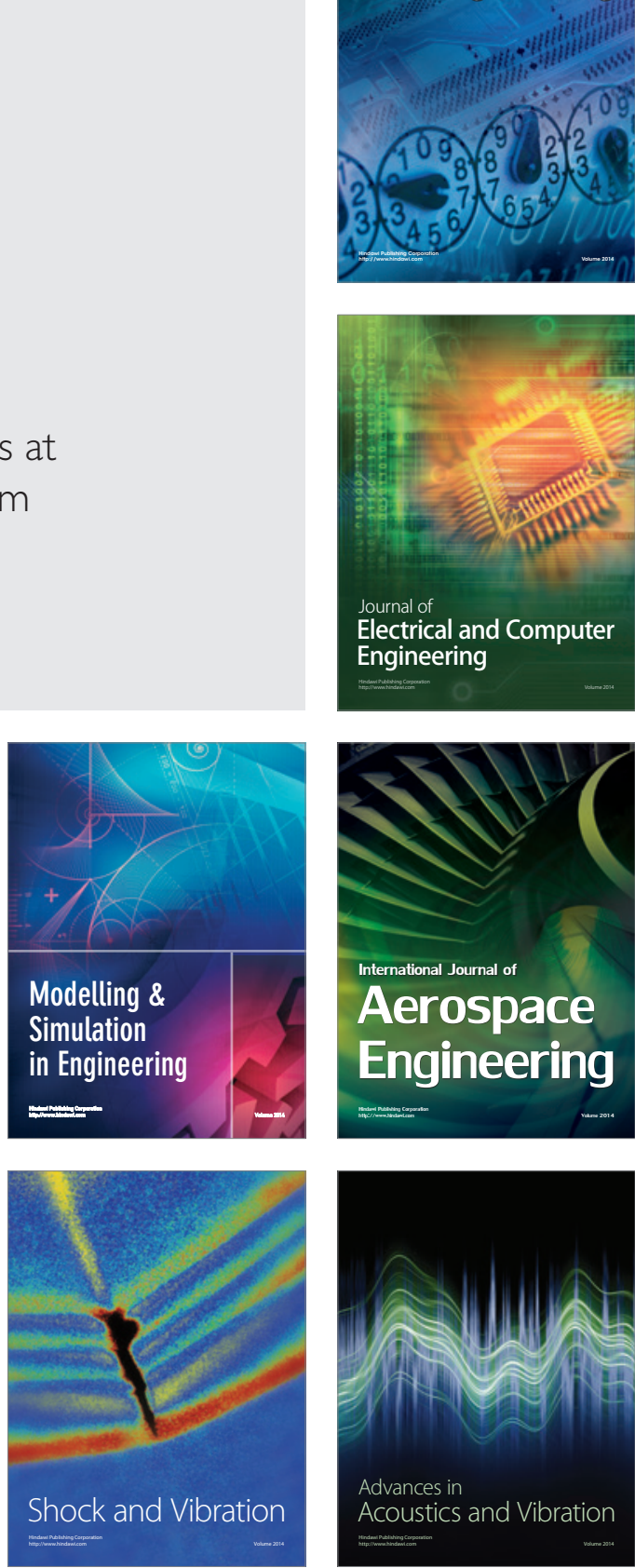